CLINICAL STUDY

\title{
Biochemical markers of bone turnover and their relation to forearm bone mineral density in persons of Pakistani and Norwegian background living in Oslo, Norway: The Oslo Health Study
}

\author{
Kristin Holvik ${ }^{1}$, Haakon E Meyer ${ }^{1,2}$, Anne Johanne Søgaard ${ }^{1,2}$, Randi Selmer ${ }^{2}$, Egil Haug $^{3}$ and Jan A Falch ${ }^{3}$ \\ ${ }^{1}$ Institute of General Practice and Community Medicine, University of Oslo, PO Box 1130 Blindern, N-O318 Oslo, Norway, ${ }^{2}$ Division of Epidemiology, \\ Norwegian Institute of Public Health, Oslo, Norway and ${ }^{3}$ Center of Endocrinology, Aker University Hospital, Oslo, Norway \\ (Correspondence should be addressed to K Holvik; Email: kristin.holvik@medisin.uio.no)
}

\begin{abstract}
Objective: To evaluate whether Pakistanis have increased bone turnover compared with ethnic Norwegians due to their high prevalence of vitamin D deficiency and secondary hyperparathyroidism, and whether the relation between bone turnover and bone mineral density (BMD) differs between Pakistanis and ethnic Norwegians.

Design: A cross-sectional, population-based study conducted in the city of Oslo in 2000-2001. Random samples of 132 community-dwelling Pakistani men and women of ages 40, 45, and 59-60 years, and 580 community-dwelling Norwegian men and women of ages 45 and 59-60 years are included in this substudy.

Methods: Venous serum samples were drawn for measurements of markers of the vitamin D endocrine system and the bone turnover markers osteocalcin (s-OC), bone alkaline phosphatase (s-bone ALP), and tartrate-resistant acid phosphatase (s-TRACP). BMD was measured at the forearm by singleenergy X-ray absorptiometry.

Results: Pakistanis had higher s-bone ALP compared with Norwegians. Mean (95\% CI) age-adjusted levels were $22.5(21.0,24.1) \mathrm{U} / \mathrm{l}$ in Pakistani men versus $19.3(18.6,20.1) \mathrm{U} / \mathrm{l}$ in Norwegian men, $P<0.0005$, and $20.3(18.4,22.1) \mathrm{U} / \mathrm{l}$ in Pakistani women versus $16.7(16.0,17.4) \mathrm{U} / \mathrm{l}$ in Norwegian women, $P=0.001$. There tended to be an inverse association between bone turnover and BMD in men and women of both ethnic groups, and it was strongest for s-bone ALP. Overall mean (95\% CI) distal BMD decrease was $-16(-20,-11) \mathrm{mg} / \mathrm{cm}^{2}$ per 1 s.D. increase in s-bone ALP $(P<0.0005)$ when adjusting for age, sex, and ethnicity.

Conclusions: Except for somewhat higher s-bone ALP levels in Pakistanis, there were only minor ethnic differences in bone turnover, despite a strikingly different prevalence of secondary hyperparathyroidism. Bone turnover was inversely associated with forearm BMD in both ethnic groups.
\end{abstract}

European Journal of Endocrinology 155 693-699

\section{Introduction}

Bone formation and resorption are coupled processes throughout adult life. The rate of bone turnover can be estimated by measuring serum or urine concentrations of proteins reflecting the bone remodeling process. These proteins can be divided into osteoblast-specific bone formation markers (e.g. osteocalcin (OC) and bone alkaline phosphatase (ALP)) and osteoclast-specific bone resorption markers (e.g. tartrate-resistant acid phosphatase (TRACP) and telopeptides of collagen cross-links) (1). With increasing age, bone formation tends to lag behind resorption, and increased bone turnover rate may lead to negative bone balance, resulting in bone loss (2). High serum levels of bone turnover markers reflect an increased turnover rate (3) and are related to rapid bone loss in untreated osteoporosis. A high bone turnover rate predicts fracture independent of BMD, but it is also associated with a lower BMD in elderly persons. The combination of low BMD and high levels of bone turnover markers is associated with a particularly high fracture risk (4).

Measurements of biochemical markers of bone turnover may complement BMD measurements, as an instantaneous, dynamic measurement of the bone turnover rate (5). Clinical use of biochemical bone turnover markers is presently established in monitoring the effectiveness of antiresorptive therapy in patients with osteoporosis, and potential use also includes prediction of rates of bone loss and fracture risk (3). 
However, clinical usefulness of biochemical bone turnover markers is still limited due to the large intra- and inter-individual variabilities (6).

Ethnicity is one of the many potential sources of biological variability, and ethnic bone turnover differences may be attributable to genetic or lifestyle differences (6).

Ethnic differences in the vitamin D endocrine system may possibly be involved. Higher bone turnover marker levels are seen alongside higher parathyroid hormone (PTH) levels (7).

Community-based studies show that African Americans have higher BMD compared with Caucasian Americans (8). Lower serum OC (s-OC) levels were found in AfricanAmerican women and men compared with Caucasian American women and men (9, 10). Serum bone ALP (s-bone ALP) levels were higher in African-American women compared with Caucasian American women in one study (9), but another study did not find this (10).

Only a few small studies have investigated bone turnover in populations from the Indian subcontinent. Pregnant Pakistani women living in Oslo had somewhat lower s-OC and higher total ALP levels when compared with pregnant Norwegian women, although not significantly different (11). Premenopausal Pakistani women living in Oslo had considerably higher serum total alkaline phosphatase levels compared with Norwegian women, but s-OC levels were similar (12). Thus, existing data are few and inconclusive.

In the Oslo Health Study, we have previously shown that in spite of a much higher prevalence of vitamin D deficiency and secondary hyperparathyroidism in Pakistan-born than in Norwegian-born persons (13), BMD of the distal forearm was at least as high in the Pakistanis as that in Norwegians (14). To explore these ethnic differences further, we studied serum levels of biochemical bone turnover markers and their relation to BMD. Our research investigates whether Pakistanis living in Oslo have a higher bone turnover rate compared with ethnic Norwegians, and whether the association between bone turnover and BMD differs between Norwegians and Pakistanis.

\section{Materials and methods}

The present vitamin D study is a supplementary project of The Oslo Health Study. This was a cross-sectional population-based multipurpose study conducted in 2000-2001, inviting all individuals in Oslo County born in 1924, 1925, 1940, 1941, 1955, 1960, and 1970 . The overall attendance rate was $46 \%$ $(n=18770)$, varying from $36 \%$ in 30 years old to $55 \%$ in $59-60$ years old individuals. The materials and methods, including respondent rates, are described in detail elsewhere (15). The following subgroups of participants were recruited for the vitamin D substudy performed in the period from May 2000 to January
2001. Individuals born in Norway (quoted as Norwegians): random samples of participants born in 1955 (45 years), 1940 (60 years), and 1925 (75 years). Individuals born in Pakistan (quoted as Pakistanis): random samples of all age groups in the Oslo Health Study. However, we excluded the oldest (75 years) and the youngest (30 years) group for the present analyses, as there were only three Pakistanis among the oldest and no Norwegians among the youngest, and as bone turnover may not have declined to adult levels at 30 years of age. Out of the 1281 Norwegians of ages 45-60 years invited to the substudy, 674 (53\%) participated, and $584(46 \%)$ had both bone densitometry performed and vitamin D metabolites in serum analyzed. Out of the 408 Pakistanis of ages 40-60 years invited to the substudy, 176 (43\%) participated, and 132 (32\%) had both bone densitometry performed and vitamin D metabolites in serum analyzed. In addition, three persons were excluded due to primary hyperparathyroidism as defined by serum intact PTH level $(\mathrm{s}-\mathrm{iPTH}) \geq 8.5 \mathrm{pmol} / \mathrm{l}$ and $\mathrm{s}-\mathrm{Ca}^{2+}>1.35 \mathrm{mmol} / \mathrm{l}$, and one person was excluded due to very low s-iPTH combined with high $\mathrm{s}-\mathrm{Ca}^{2+}(\mathrm{iPTH}<2.0 \mathrm{pmol} / \mathrm{l}$ and $\left.\mathrm{Ca}^{2+}>1.35 \mathrm{mmol} / \mathrm{l}\right)$, which possibly indicates malignancy.

Thus, the final sample consisted of 79 Pakistani men and 53 Pakistani women aged 40, 45, and 59-60 years, and 302 Norwegian men and 278 Norwegian women aged 45 and 59-60 years; in total 712 subjects. The number of subjects in the analyses varies due to missing data.

\section{Data collection}

All participants underwent a clinical examination, and information about various diseases, symptoms, and lifestyle factors was obtained from self-administered questionnaires (16). In addition, BMD was measured, and a non-fasting blood sample was collected from each participant on the day of attendance.

\section{Bone mineral density measurement}

$\mathrm{BMD}$ was measured at the forearm with single-energy X-ray absorptiometry (DTX-100; Osteometer MediTech Inc., Hawthorne, CA, USA). As explained in detail elsewhere (17), bone densitometry was performed at the distal (10-20\% trabecular bone) and ultradistal (50-70\% trabecular bone) forearm sites. This was done at the non-dominant forearm except in $1-2 \%$ of the cases measured at the dominant forearm where the non-dominant was ineligible. Daily calibration was carried out with a phantom provided by the manufacturer. All scans were reviewed and, if necessary, reanalyzed. Subjects who did not have a valid bone densitometry measurement were excluded from the data analyses that involved BMD. 


\section{Blood sample analyses}

The serum samples were first stored at $-20{ }^{\circ} \mathrm{C}$ for up to 8 weeks at the screening station, and then kept frozen at $-70{ }^{\circ} \mathrm{C}$ until analyzed in the Hormone Laboratory, Aker University Hospital. Assays for serum 25-hydroxyvitamin D levels (s-25(OH)D) and s-iPTH were performed as previously described (13).

Serum osteocalcin was measured by competitive luminoimmunoassay (B R A H M S Diagnostica GMBH, Berlin, Germany). The intra- and interassay coefficients of variation (CVs) for s-OC were 5-10 and $13-19 \%$ respectively.

Serum bone alkaline phosphatase (s-bone ALP) was measured by enzyme activity assessment after immune extraction (Metra Biosystems Inc., Mountain View, CA, USA). The intra- and interassay CVs for s-bone ALP were $2-6$ and $11-12 \%$ respectively.

Serum tartrate-resistant acid phosphatase (s-TRACP) was measured by enzyme activity assessment after immune extraction (Suomen Bioanalytiikka Oy, Oulu, Finland). This assay measures the active isoform $5 \mathrm{~b}$ derived from osteoclasts. The intra- and interassay CVs for s-TRACP were 5-12 and 8-14\% respectively.

\section{Data analysis}

Statistical tests were performed using the software SPSS 11.0 for Windows. Ethnic differences in bone turnover marker levels and the relationship between bone turnover marker levels and BMD were assessed by one-way ANOVA and linear regression analysis. The association between s-iPTH and bone turnover marker levels was assessed by partial correlations. The analyses are not adjusted for time since last meal, as additional analyses showed that such adjustment had negligible influence on s-iPTH and s-25(OH)D levels. Potential statistical interaction between ethnicity and s-iPTH on the effect of bone turnover marker levels was evaluated by including an interaction term in linear regression analysis. As the sample of Pakistanis was younger than the Norwegians (Table 1), the statistical analyses were age-adjusted, as shown in tables. As estrogen therapy is known to influence bone metabolism, and varies between ethnic groups (Table 1), we also included an adjustment for this, although we acknowledge that we have a mixed age sample and estrogen therapy is predominantly used by the 60-year olds in our sample. Additional data analyses were performed stratified on ethnicity, sex, and age.

\section{Ethics}

The study protocol was reviewed by the Regional Committee for Medical Research Ethics and approved by the Norwegian Data Inspectorate. Written informed consent was collected from all participants.

\section{Results}

\section{Serum levels of biochemical markers of bone turnover}

Background characteristics of the sample are shown in Table 1. Overall unadjusted mean ( \pm s.D.) serum levels of s-OC, s-bone ALP, and s-TRACP were $1.3( \pm 0.5)$ $\mathrm{nmol} / \mathrm{l}(n=710), 18.7( \pm 6.3) \mathrm{U} / \mathrm{l}(n=694)$, and 2.51 $( \pm 0.68) \mathrm{U} / \mathrm{l}(n=690)$ respectively. s-OC ranged from 0.2 to $4.1 \mathrm{nmol} / \mathrm{l}$, s-bone ALP ranged from 4.2 to $58.9 \mathrm{U} / \mathrm{l}$, and s-TRACP ranged from 1.06 to $6.45 \mathrm{U} / \mathrm{l}$. Only seven subjects (1.0\%) had s-OC above the method's reference range and five $(0.7 \%)$ had s-bone ALP above the method's reference range.

As previously shown (13), the Pakistanis had considerably lower s-25(OH)D and higher s-iPTH compared with the Norwegians. In spite of this, distal and ultradistal BMD did not differ between the ethnic groups. These results are presented in Table 2, together

Table 1 Background characteristics of Pakistanis and Norwegians attending the vitamin D substudy of the Oslo Health Study.

\begin{tabular}{|c|c|c|c|c|c|c|}
\hline & \multicolumn{3}{|c|}{ Men } & \multicolumn{3}{|c|}{ Women } \\
\hline & $\begin{array}{c}\text { Norwegians } \\
n=302\end{array}$ & $\begin{array}{c}\text { Pakistanis } \\
n=79\end{array}$ & $\begin{array}{c}P \\
{\left(\text { (ethnicity) }^{a}\right.}^{a}\end{array}$ & $\begin{array}{c}\text { Norwegians } \\
n=278\end{array}$ & $\begin{array}{c}\text { Pakistanis } \\
n=53\end{array}$ & $\begin{array}{c}P \\
{(\text { ethnicity })^{a}}^{a}\end{array}$ \\
\hline \multicolumn{7}{|l|}{ Age $(n)$} \\
\hline 40 & - & 34 & - & - & 27 & - \\
\hline 45 & 76 & 21 & - & 91 & 19 & - \\
\hline $59 / 60$ & 226 & 24 & - & 187 & 7 & - \\
\hline $\begin{array}{l}\text { Mean }(95 \% \mathrm{Cl}) \text { body mass index } \\
\left(\mathrm{kg} / \mathrm{m}^{2}\right)\end{array}$ & $\begin{array}{c}26.6 \\
(26.2,27.0)\end{array}$ & $\begin{array}{c}27.6 \\
(26.8,28.3)\end{array}$ & 0.026 & $\begin{array}{c}25.0 \\
(24.5,25.5)\end{array}$ & $\begin{array}{c}30.4 \\
(29.3,31.6)\end{array}$ & $<0.0005$ \\
\hline$\%$ Present smokers & $31 \%$ & $32 \%$ & 0.89 & $30 \%$ & $6 \%$ & 0.001 \\
\hline$\%$ Present estrogen therapy users & - & - & - & $32 \%$ & $2 \%$ & $<0.0005$ \\
\hline$\%$ Present bisphosphonate users & 0 & 0 & - & $1 \%$ & 0 & 0.59 \\
\hline$\%$ Calcium supplement users & $3 \%$ & 0 & 0.37 & $16 \%$ & $4 \%$ & 0.028 \\
\hline $\begin{array}{l}\text { Mean }(95 \% \mathrm{Cl}) \text { daily vitamin } \mathrm{D} \\
\text { intake from supplements }(\mu \mathrm{q})\end{array}$ & $\begin{array}{c}4.4 \\
(3.9,5.0)\end{array}$ & $\begin{array}{c}2.3 \\
(1.0,3.5)\end{array}$ & 0.002 & $\begin{array}{c}5.4 \\
(4.8,6.0)\end{array}$ & $\begin{array}{l}2.6 \\
(0.9,4.2)\end{array}$ & 0.002 \\
\hline
\end{tabular}

$\mathrm{Cl}$, confidence interval.

${ }^{a}$ Means are compared within the genders by ANOVA; percentages are compared within the genders by $\chi^{2}$-test or Fisher's exact test. 
Table 2 Age-adjusted $^{\mathrm{a}}$ mean $(95 \% \mathrm{Cl})$ serum 25-hydroxyvitamin $\mathrm{D}(\mathrm{s}-25(\mathrm{OH}) \mathrm{D})$ levels, serum intact parathyroid hormone (s-iPTH) levels, bone mineral density (BMD), and serum bone turnover marker levels according to sex and ethnic background.

\begin{tabular}{|c|c|c|c|c|c|c|}
\hline & \multicolumn{3}{|c|}{ Men } & \multicolumn{3}{|c|}{ Women } \\
\hline & $\begin{array}{c}\text { Norwegians } \\
n=302\end{array}$ & $\begin{array}{c}\text { Pakistanis } \\
n=79\end{array}$ & $\begin{array}{l}P \text { (ethnicity) } \\
\text { ANOVA }\end{array}$ & $\begin{array}{c}\text { Norwegians } \\
n=278\end{array}$ & $\begin{array}{c}\text { Pakistanis } \\
n=53\end{array}$ & $\begin{array}{c}P \text { (ethnicity), } \\
\text { ANOVA }\end{array}$ \\
\hline s-25(OH)D (nmol/l) & $74.1(71.5,76.7)$ & $29.4(24.0,34.9)$ & $<0.0005$ & $75.9(73.1,78.7)$ & $24.0(17.1,31.0)$ & $<0.0005$ \\
\hline s-iPTH (pmol/l) & $5.33(5.06,5.61)$ & $6.36(5.78,6.94)$ & 0.003 & $5.10(4.79,5.41)$ & $7.63(6.85,8.42)$ & $<0.0005$ \\
\hline Distal BMD $\left(\mathrm{mg} / \mathrm{cm}^{2}\right)$ & $539(532,545)$ & $541(527,555)$ & 0.74 & $440(434,446)$ & $431(415,447)$ & 0.29 \\
\hline Ultradistal BMD $\left(\mathrm{mg} / \mathrm{cm}^{2}\right)$ & $446(439,453)$ & $451(436,466)$ & 0.59 & $345(338,351)$ & $339(323,356)$ & 0.56 \\
\hline \multicolumn{7}{|l|}{ Bone turnover markers } \\
\hline $\mathrm{s}-\mathrm{OC}(\mathrm{nmol} / \mathrm{l})$ & $1.4(1.3,1.4)$ & $1.3(1.2,1.4)$ & 0.71 & $1.4(1.3,1.4)$ & $1.2(1.0,1.4)$ & 0.10 \\
\hline s-bone ALP (U/I) & $19.3(18.6,20.1)$ & $22.5(21.0,24.1)$ & $<0.0005$ & $16.7(16.0,17.4)$ & $20.3(18.4,22.1)$ & 0.001 \\
\hline s-TRACP (U/I) & $2.54(2.46,2.61)$ & $2.50(2.33,2.66)$ & 0.71 & $2.48(2.40,2.57)$ & $2.49(2.28,2.71)$ & 0.93 \\
\hline
\end{tabular}

s-OC, serum osteocalcin; s-bone ALP, serum bone alkaline phosphatase; s-TRACP, serum tartrate-resistant acid phosphatase.

${ }^{a}$ Adjusted at different mean ages for men and women.

with our new findings. Regarding biochemical markers of bone turnover, s-OC was similar in Norwegian and Pakistani men (Table 2). Pakistani women had slightly lower $(0.2 \mathrm{nmol} / \mathrm{l}) \mathrm{s}-\mathrm{OC}$ than Norwegian women, a difference that became statistically significant when adjusting for estrogen therapy (Table 3). Norwegian women were by far the most frequent estrogen users (Table 1). Further adjustment for vitamin D status increased the ethnic difference in s-OC in women.

In both men and women, mean s-bone ALP levels were higher in Pakistanis than in Norwegians (Table 2). The ethnic difference was in the magnitude of $3 \mathrm{U} / \mathrm{l}$, corresponding to $1 / 2$ s.D. When we stratified for age, the ethnic s-bone ALP difference was larger for the oldest $(\Delta=3.8(1.4,6.2) \mathrm{U} / \mathrm{l}$ for men $59-60$ years and $\Delta=3.0$ $(0.5,5.6) \mathrm{U} / \mathrm{l}$ for men $40-45$ years, both were significant; $\Delta=9.7(4.4,15.1) \mathrm{U} / \mathrm{l}$ for women $59-60$ years and $\Delta=1.7(0.1,3.3) \mathrm{U} / \mathrm{l}$ for women $40-45$ years, both were significant).

Introducing an adjustment for the possible smaller skeletal size of Pakistanis by dividing distal BMD by distal area did not alter the results substantially, although it increased the ethnic s-bone ALP difference somewhat (mean $(95 \% \mathrm{CI})$ ethnic s-bone ALP difference 4.0
$(2.1,5.8) \mathrm{U} / \mathrm{l}$ in men and $4.4(2.4,6.4) \mathrm{U} / \mathrm{l}$ in women). When we controlled for estrogen therapy in women, the ethnic s-bone ALP difference was still present but slightly weaker (Table 3). The ethnic s-bone ALP difference was still much stronger in the oldest after adjustment for estrogen therapy, but attenuated in both age strata $(\Delta=8.0(3.4,12.7) \mathrm{U} / \mathrm{l}$ in $59-60$ years old women and $\Delta=1.5(-0.2,3.1) \mathrm{U} / \mathrm{l}$ in $40-45$ years old women). When we introduced an additional adjustment for vitamin D status, the effect of ethnicity vanished in women, but was only slightly attenuated in men.

Three Pakistani men had s-bone ALP levels of above $44 \mathrm{U} / \mathrm{l}$, suggesting a disturbed bone metabolism. Excluding these lead to an attenuation of the ethnic s-bone ALP difference in men (age-adjusted mean 21.3 $(19.9,22.6) \mathrm{U} / \mathrm{l}$ for Pakistani men; age-adjusted ethnic difference $1.9(0.3,3.5) \mathrm{U} / \mathrm{l})$.

We observed no ethnic differences in s-TRACP levels, and statistical adjustment did not alter this (Table 3).

Performing additional analyses with adjustment for calcium supplementation or excluding the three bisphosphonate users led to negligible alterations in the observed ethnic differences in bone turnover marker levels.

Table 3 Mean ethnic differences $(95 \% \mathrm{Cl}$ ) between Norwegians and Pakistanis in bone turnover markers, adjusted for confounders (PakistanisNorwegians).

\begin{tabular}{|c|c|c|c|c|}
\hline & $n$ & $\Delta^{a}$ & $\Delta^{\mathrm{b}}$ & $\Delta^{\mathrm{c}}$ \\
\hline \multicolumn{5}{|l|}{ Men } \\
\hline $\mathrm{s}-\mathrm{OC}(\mathrm{nmol} / \mathrm{l})$ & 379 & $0(-0.2,0.1)$ & - & $0(-0.2,0.2)$ \\
\hline s-bone ALP (U/I) & 372 & $3.2(1.4,5.0)$ & - & $2.6(0.5,4.8)$ \\
\hline s-TRACP (U/I) & 368 & $-0.07(-0.26,0.13)$ & - & $0.13(-0.11,0.37)$ \\
\hline \multicolumn{5}{|l|}{ Women } \\
\hline $\mathrm{s}-\mathrm{OC}(\mathrm{nmol} / \mathrm{l})$ & 331 & $-0.2(-0.4,0)$ & $-0.2(-0.4,0)$ & $-0.3(-0.5,0)$ \\
\hline s-bone ALP (U/I) & 322 & $3.6(1.5,5.6)$ & $3.0(1.2,4.9)$ & $0.6(-1.6,2.8)$ \\
\hline s-TRACP (U/I) & 322 & $0.04(-0.21,0.29)$ & $-0.02(-0.25,0.21)$ & $-0.08(-0.37,0.21)$ \\
\hline
\end{tabular}

s-OC, serum osteocalcin; s-bone ALP, serum bone alkaline phosphatase; s-TRACP, serum tartrate-resistant acid phosphatase.

${ }^{a}$ Mean differences adjusted for age.

${ }^{\mathrm{b}}$ Mean differences adjusted for age and present estrogen therapy.

cMean differences adjusted for age (and present estrogen therapy in women) and s-25(OH)D and s-iPTH. 


\section{Correlation between s-iPTH and bone turnover markers}

We found no statistical interaction between the effects of ethnicity and s-iPTH levels on bone turnover marker levels, suggesting a similar association in Norwegians and Pakistanis. In women, age- and ethnicity-adjusted correlation analyses showed a weak positive correlation between s-iPTH and s-OC $(r=0.11, P=0.046)$ and a stronger positive correlation between s-iPTH and s-bone ALP $(r=0.24, P<0.0005)$. s-iPTH levels seemed to explain s-bone ALP levels identical in Pakistani and Norwegian women (data not shown). In women, there was no correlation between s-iPTH and s-TRACP. In men, we found no significant correlations between s-iPTH and any bone turnover marker.

\section{Association between bone turnover marker levels and BMD}

Distal BMD decreased significantly with increasing s-OC only in Norwegian women (Table 4), although the same tendency was present in Norwegian and Pakistani men. There was a similar relationship between s-OC and ultradistal BMD, which was statistically significant also in Norwegian men. Both distal and ultradistal BMD decreased significantly with increasing s-bone ALP in all groups. Distal and ultradistal BMD decreased with increasing s-TRACP in Pakistani men and Norwegian women. This tendency was also observed in Pakistani women, although it was not statistically significant.

\section{Discussion}

In spite of the large ethnic difference in s-25(OH)D and s-iPTH, we found only small differences in bone turnover between Norwegians and Pakistanis living in Oslo, with the exception of s-bone ALP, which was about $3 \mathrm{U} / \mathrm{l}$ (approximately 1/2 S.D.) higher in Pakistanis of both sexes. We also found an inverse association between bone turnover and forearm BMD in both ethnic groups, but this association was most pronounced in Norwegian women. S-bone ALP showed the most consistent association with BMD. In women, this relationship was in the magnitude of 1/3-1/4 s.D. decrease in BMD per 1 s.D. increase in s-bone ALP.

\section{Ethnic differences in bone turnover marker levels}

Most studies investigating ethnic bone turnover differences have compared African-American and Western Caucasian populations, and very few data on immigrants from the Indian subcontinent exist. Unlike our study, one study found that premenopausal Indian/Pakistani women living in the US with low vitamin D status $(n=47)$ had much higher urinary bone resorption marker levels (N-Tx) but similar s-bone ALP compared with American Caucasian women with normal vitamin D status $(n=47)(18)$. Another small study found lower s-OC accompanying a lower vitamin D status in healthy young adult Asian Indians living in the US $(n=18)$ when compared with Caucasian Americans $(n=27)(19)$, which is in agreement with our results. However, ethnicity was not a significant determinant of s-OC after adjustment for age and sex.

Our results are also in agreement with previous data from Oslo, where Pakistani premenopausal women had lower s-25(OH)D and higher s-iPTH levels but similar BMD at all sites measured by dual energy X-ray absorptiometry when compared with Norwegian women (12). This study reported total (but not bonespecific) ALP levels, which were considerably higher in the Pakistani women (187 vs $131 \mathrm{U} / \mathrm{l}, \mathrm{P}<0.001)$.

Data from Pakistan, analyzed in the same laboratory as our blood samples, showed that mean s-bone ALP was around $20 \mathrm{U} / \mathrm{l}$ in healthy first-time delivering Pakistani women in Karachi, which is of the same magnitude as in the Pakistani women in our data (20).

A higher s-bone ALP in the Pakistanis may be due to different ethnic-specific normal ranges (6), or it may imply a secondary hyperparathyroidism-induced

Table 4 Age-adjusted mean $\left(95 \% \mathrm{Cl}\right.$ ) difference in bone mineral density (BMD; $\mathrm{mg} / \mathrm{cm}^{2}$ ) per 1 S.D. ${ }^{\mathrm{a}}$ increase in serum levels of osteocalcin (s-OC), bone alkaline phosphatase (s-bone ALP), or tartrate-resistant acid phosphatase (s-TRACP).

\begin{tabular}{|c|c|c|c|c|}
\hline & \multicolumn{2}{|c|}{ Men } & \multicolumn{2}{|c|}{ Women } \\
\hline & Norwegians $n=294$ & Pakistanis $n=77$ & Norwegians $n=272$ & Pakistanis $n=49$ \\
\hline \multicolumn{5}{|l|}{$\mathrm{s}-\mathrm{OC}$} \\
\hline Distal BMD $\left(\mathrm{mg} / \mathrm{cm}^{2}\right)$ & $-4(-11,4) P=0.32$ & $-10(-23,2) P=0.10$ & $-15(-20,-10) P<0.0005$ & $8(-9,25) P=0.34$ \\
\hline $\begin{array}{l}\text { Ultradistal BMD }\left(\mathrm{mg} / \mathrm{cm}^{2}\right) \\
\text { s-bone ALP }\end{array}$ & $-10(-18,-1) P=0.020$ & $-10(-23,4) P=0.17$ & $-15(-20,-9) P<0.0005$ & $1(-16,19) P=0.88$ \\
\hline Distal BMD $\left(\mathrm{mg} / \mathrm{cm}^{2}\right)$ & $-11(-19,-4) P=0.004$ & $-12(-20,-4) P=0.003$ & $-20(-26,-14) P<0.0005$ & $-20(-36,-5) P=0.013$ \\
\hline $\begin{array}{l}\text { Ultradistal BMD }\left(\mathrm{mg} / \mathrm{cm}^{2}\right) \\
\text { s-TRACP }\end{array}$ & $-12(-20,-3) P=0.008$ & $-12(-20,-3) P=0.011$ & $-21(-27,-14) P<0.0005$ & $-21(-36,-5) P=0.009$ \\
\hline Distal BMD $\left(\mathrm{mg} / \mathrm{cm}^{2}\right)$ & $1(-5,8) P=0.68$ & $-21(-33,-10) P=0.001$ & $-11(-17,-6) P<0.0005$ & $-4(-20,13) P=0.65$ \\
\hline Ultradistal BMD $\left(\mathrm{mg} / \mathrm{cm}^{2}\right)$ & $1(-7,8) P=0.84$ & $-22(-35,-9) P=0.001$ & $-11(-17,-5) P<0.001$ & $-5(-22,12) P=0.57$ \\
\hline
\end{tabular}

\footnotetext{
${ }^{a_{1}}$ s.D. s-osteocalcin corresponds to $0.5 \mathrm{nmol} / /$; 1 s.D. s-bone ALP corresponds to $6.3 \mathrm{U} / \mathrm{l} ; 1$ s.D. s-TRACP corresponds to $0.68 \mathrm{U} / \mathrm{l}$.
} 
increase in bone turnover in the Pakistanis, which may in turn involve an increased bone loss. This notion is not supported by our findings, as we observed similar forearm BMD in the Pakistanis and the Norwegians. However, measurements of biochemical bone turnover markers are an instantaneous, dynamic measurement of the rate of bone turnover, which changes more rapidly than BMD and thereby precedes BMD changes (5).

An explanation for the higher s-bone ALP level but not a higher s-OC level in Pakistanis may be that these two bone formation markers are released into the circulation at different stages of the osteoblast development, s-bone ALP being elevated in the osteoid formation phase and s-OC being elevated in the mineralization phase. In the presence of a mineralization defect, the Pakistanis may have increased osteoid formation, characterized by the high s-bone ALP levels; however, this may not be accompanied by increased mineralization (21).

\section{Association between bone turnover and BMD}

We observed a consistent trend of decreasing BMD with increasing serum bone turnover marker levels in men and women of both ethnic groups, except for s-OC in Pakistani women and s-TRACP in Norwegian men. S-bone ALP showed the strongest and most consistent association with BMD. Thus, bone turnover markers predict forearm BMD on a group level in both Pakistanis and Norwegians. A US population-based study also found an inverse association between s-OC respectively s-bone ALP and BMD at various sites in women (22). In that study, forearm BMD correlated inversely with these two bone turnover markers in postmenopausal women with and without estrogen treatment, and the association was stronger for s-bone ALP. However, in premenopausal women, forearm BMD did not correlate with s-OC or s-bone ALP levels in that study. This is also in agreement with our data (not shown).

\section{Methodological considerations and implications}

The most pronounced associations between bone turnover markers and BMD were observed in Norwegian women. Caucasian women are the most commonly studied group and established as a high-risk population. Our sample of Pakistanis was smaller than the sample of Norwegians, and they were predominantly premenopausal. For the sake of completeness, we also included the seven Pakistani women of ages 59-60 years who participated, although we acknowledge that they were few. Although our results do not support an increased bone turnover or reduced BMD in the Pakistanis compared with ethnic Norwegians, their very low vitamin D status and prevalent secondary hyperparathyroidism may be expected to be as harmful for their bone as in Caucasian women. Our data are merely cross-sectional, and the Pakistanis who presently live in Oslo may still be at an age where they are protected by endogenous estrogens. It would have been desirable to include more elderly Pakistani women, as the negative effect of secondary hyperparathyroidism on $\mathrm{BMD}$ is expected to deteriorate in the absence of estrogens after menopause. This is also suggested by the higher s-bone ALP in the 59-60 years old Pakistani women. A follow-up study would be needed to determine the rate of bone loss.

Due to the low attendance rate, we cannot rule out the possibility that the sample of Pakistanis who attended the Oslo Health Study may constitute a selected group who are similar to Norwegians with regard to lifestyle and therefore also with regard to bone turnover and BMD. Thus, we may have failed to demonstrate any ethnic differences in these variables. However, a larger proportion of persons receiving disability benefit participated among non-western immigrants than among ethnic Norwegians, indicating that the non-western immigrants who participated could be expected to be unhealthier than the ethnic Norwegians who participated (15). Moreover, the very low s-25(OH)D and high s-iPTH levels in the Pakistani individuals imply already interesting biochemical ethnic differences which would be expected to be of great importance for their bone remodeling. Thus, we may speculate that the Pakistanis have an altered handling of vitamin D metabolism, protecting their skeleton against excessive resorption despite their low vitamin D supply.

\section{Conclusion}

We conclude that the ethnic differences in bone turnover expressed by serum levels of biochemical markers of bone turnover are not as large as the difference in prevalence of vitamin D deficiency and secondary hyperparathyroidism should suggest, and that bone turnover is inversely associated with forearm BMD in both Norwegians and Pakistanis.

\section{Acknowledgements}

The data collection was conducted as part of the Oslo Health Study carried out in 2000-2001 as a collaboration between the National Health Screening Service (now the Norwegian Institute of Public Health), the University of Oslo, and the Municipality of Oslo. The project has been financed with the aid of EXTRA funds from the Norwegian Foundation for Health and Rehabilitation.

\section{References}

1 Calvo MS, Eyre DR \& Gundberg CM. Molecular basis and clinical application of biological markers of bone turnover. Endocrine Reviews 199617 333-368. 
2 Rosen CJ \& Tenenhouse A. Biochemical markers of bone turnover A look at laboratory tests that reflect bone status. Postgraduate Medicine $1998 \mathbf{1 0 4} 101-110,113$.

3 Hannon RA \& Eastell R. Biochemical markers of bone turnover and fracture prediction. Journal of the British Menopause Society 20039 10-15.

4 Garnero P, Hausherr E, Chapuy MC, Marcelli C, Grandjean H, Muller C, Cormier C, Breart G, Meunier PJ \& Delmas PD. Markers of bone resorption predict hip fracture in elderly women: The EPIDOS Prospective Study. Journal of Bone and Mineral Research 199611 1531-1538.

5 Khosla S \& Kleerekoper M. Biochemical markers of bone turnover. In Primer on the Metabolic Bone Diseases and Disorders of Mineral Metabolism. edn 5, ch 26 pp 166-173. Ed. MJ Favus, Washington, DC: American Society for Bone and Mineral Research, 2003.

6 Hannon R \& Eastell R. Preanalytical variability of biochemical markers of bone turnover. Osteoporos International 200011 (Suppl 6) S30-S44.

7 Gallagher JC, Kinyamu HK, Fowler SE, Dawson-Hughes B, Dalsky GP \& Sherman SS. Calciotropic hormones and bone markers in the elderly. Journal of Bone and Mineral Research 199813 475-482.

8 Harris SS, Wood MJ \& Dawson-Hughes B. Bone mineral density of the total body and forearm in premenopausal black and white women. Bone 199516311 S-315S.

9 Gundberg CM, Looker AC, Nieman SD \& Calvo MS. Patterns of osteocalcin and bone specific alkaline phosphatase by age, gender, and race or ethnicity. Bone 200231 703-708.

10 Han ZH, Palnitkar S, Rao DS, Nelson D \& Parfitt AM. Effects of ethnicity and age or menopause on the remodeling and turnover of iliac bone: implications for mechanisms of bone loss. Journal of Bone and Mineral Research 199712 498-508.

11 Brunvand L \& Haug E. Vitamin D deficiency amongst Pakistani women in Oslo. Acta Obstetricia et Gynecologica Scandinavica 1993 72 264-268.

12 Falch JA \& Steihaug S. Vitamin D deficiency in Pakistani premenopausal women living in Norway is not associated with evidence of reduced skeletal strength. Scandinavian Journal of Clinical and Laboratory Investigation 2000 60 103-109.

13 Meyer HE, Falch JA, Søgaard AJ \& Haug E. Vitamin D deficiency and secondary hyperparathyroidism and the association with bone mineral density in persons with Pakistani and Norwegian background living in Oslo, Norway. The Oslo Health Study. Bone $200435412-417$.

14 Alver K, Meyer HE, Falch JA \& Søgaard AJ. Bone mineral density in ethnic Norwegians and Pakistani immigrants living in Oslo - The Oslo Health Study. Osteoporosis International 200516 623-630.

15 Søgaard AJ, Selmer R, Bjertness E \& Thelle D. The Oslo Health Study: the impact of self-selection in a large, population-based survey. International Journal for Equity in Health 200433.

16 Norwegian Institute of Public Health website. http://www.fhi.no/ artikler/?id = 544642006 .

17 Berntsen GK, Fønnebø V, Tollan A, Søgaard AJ \& Magnus JH. Forearm bone mineral density by age in 7620 men and women: the Tromsø study, a population-based study. American Journal of Epidemiology $2001 \mathbf{1 5 3}$ 465-473.

18 Alekel DL, Mortillaro E, Hussain EA, West B, Ahmed N, Peterson CT, Werner RK, Arjmandi BH \& Kukreja SC. Lifestyle and biologic contributors to proximal femur bone mineral density and hip axis length in two distinct ethnic groups of premenopausal women. Osteoporosis International $19999327-338$.

19 Awumey EM, Mitra DA, Hollis BW, Kumar R \& Bell NH. Vitamin D metabolism is altered in Asian Indians in the southern United States: a clinical research center study. Journal of Clinical Endocrinology and Metabolism 199883 169-173.

20 Brunvand L, Shah SS, Bergström S \& Haug E. Vitamin D deficiency in pregnancy is not associated with obstructed labor. A study among Pakistani women in Karachi. Acta Obstetricia et Gynecologica Scandinavica 199877 303-306.

21 Daniels ED, Pettifor JM \& Moodley GP. Serum osteocalcin has limited usefulness as a diagnostic marker for rickets. European Journal of Pediatrics 2000159 730-733.

22 Melton LJ III, Khosla S, Atkinson EJ, O’Fallon WM \& Riggs BL. Relationship of bone turnover to bone density and fractures. Journal of Bone and Mineral Research 199712 1083-1091.

Received 29 June 2006

Accepted 29 August 2006 